\title{
Acetato de Ciproterona en Hipersexualidad Masculina.
}

I) F. Fermande Cardona Arango

\section{INTRODUCCION}

Existe en la consulta del ginecólogo-endocrinólogo una patología que tiene una frecuencia mucho menor que la ocasionada por la frigidez, pero no por ello deja de ser de igual importancia, y son los problemas de una hipersexualidad que comúnmente llevan a un comportamiento sexual anormal en el sexo femenino y que también pueden afectar al sexo masculino y producir en el otro sexo serios conflictos cuando existen relaciones sexuales en uniones estables. Tanto el hipotálamo masculino como el femenino pueden tener una receptivilidad anormal a las hormonas masculinas y desencadenar allí estos trastornos de hipersexualidad. En esta comunicación se presentará un caso masculino, pero se quiere dejar establecido que esta patología en su modalidad respectiva, se puede presentar en el sexo femenino.

\section{MATERIAL Y METODOS}

Se estudia un paciente de 31 años, con buen nivel cultural e intelectual y con estudios universitarios completos que trabaja como educador y orientador en el sentido de ilustración y formación para lograr una comunidad más sana y equilibrada, desarrollando su labor en todos los niveles, es decir, trabajando con personal humano de uno y otro sexo y desde la niñez hasta la senectud.

Se hacen dosificaciones hormonales de 17 cetoesteroides totales (4) y fraccionados por cromotografía en columna (3), se procede a un tratamiento con prednisona, posteriormente reforzado con reserpina y luego fenotiazinas, sustituídas, en otra etapa

Profesor III Dpto. de Obstetricia y Ginecología.

Facultad de medicina. Universidad de Antioquia, Medellín. Colombia. con prodnisona y estrógenos conjugados y luego cambiando estos últimos por 6 metil 17 acetoxi-progesterona y finalmente con solo acetato de ciproterona como anti-andrógeno, se comenta la evolución y los resultados obtenidos.

\section{HALLAZGOS}

Paciente de 31 años que consulta por una hiperactividad sexual consistente en onanismo muy frecuente y relaciones homosexuales con adultos y en múltiples ocasiones con pedofilia sin pederastia. Se procede a una dosificación de 17-cetoesteroides en la diuresis de 24 horas que indica una cifra de 16.5 miligramos, 1.5 miligramos por encima de la media hora para su sexo y edad. Se efectua una dosificación de 17-cetoesteroides fraccionados por cromatografía en columna de óxido de alumino activado, con la técnica de Dingemanse y se encuentran unas cifras expresadas en miligramos de: I. Androsterona 2.679 (1910/o de lo normal. II. Androstenolona 1.689 (231\%/o de lo normal). III. Dehidroepiandrosterona $1.448\left(128^{\circ} / 0\right.$ de lo normal). IV. Androsterona 3.787 ( $71 \%$ o de lo normal). V. Etiocolanolona $4.039(1080 / 0$ de lo normal). VI. Hidroxiandrosterona 1.717 (257 $0 / 0$ de lo normal). VII. Hidroxieticolanolona $0.632(73 \%$ o de lo normal $)$. VIII. 17-cetoesteroides no identificados $0.758\left(67^{\circ} / \mathrm{o}\right.$ de lo normal).

El estudio en conjunto revela un aumento de los andrógenos suprarrenales en unas cifras ligeramente disminuidas de la androsterona que es de origen gonadal. Se procede en vista de ello y basados en otras experiencias positivas (2) a una terapia con prednisona a la dosis de 5 miligramos a mañana y tarde para poner la suprarrenal en un relati- 
vo reposo de la esteroidegenesis androgénica y se observa la evolución del paciente cada dos meses durante un año. No se encuentra un cambio significativo en su conducta y su problema de hiperactividad sexual continúa.

Se hace una dosificación control de los 17 cetoesteroides totales y se obtiene una cifra de 11.5 mgrs. en la diuresis de 24 horas. Se continúa la terapia con prednisona y buscando una disminución de su líbido se agrega una dosis de 0.25 mgrs. de reserpina diaria, se continúa observando cada dos meses y al completar el semestre, como no se observa modificación se decide duplicar la dosis de reserpina a 0.25 miligramos cada 12 horas. Al completar el año sin cambio, se agrega a todo lo anterior que se continúe en igual dosis con fenotiazidas sustituidas, como la perfenazina para potencializar la reserpina y como droga psico-sedante con 1 mgr. por día. En los 6 meses siguientes no se observan cambios y se aumenta la dosis de prednisona a 15 miligramos diarios sin modificar la terapia complementaria; se observa al cabo de 6 meses un aumento de peso y una tendencia en los rasgos faciales hacia el tipo Cushing, sin cambios en su comportamiento sexual. En vista de ello se baja la dosis de prednisona en forma gradual a 5 miligramos día y se aumenta la reserpina a un miligramo día, se continúa la observación y se aprecian cambios físicos regresivos por el descenso de la prednisona pero la hipersexualidad permanece igual en los siguientes 6 meses. Al completar este tiempo de tratamiento sin resultados se intenta en forma experimental y buscando un efecto antagónico, es decir una acción antiandrógena, una terapia con 5 miligramos diarios de prednisona y 0.065 mgr. de estrógenos conjugados interdiarios por otros 6 meses al cabo de los cuales se suspenden los estr-ogenos por resultado negativo pero sin ginecomastia. Ante el fracaso de todo lo anterior se inicia una prueba terapéutica con 5 mgr. por día de prednisona y 150 mgr cada dos meses de 17 acetoxiprogesterona, intentando con ellos bloquear los factores liberadores hipotalámicos de RH FSH y R.H.L.H. Al cabo de un año se suspenden los progestágenos por persistir la hipersexualidad; teniendo en este momento la seguridad de poder iniciar en un futuro próximo de 6 meses una terapia con acetato de ciproterona se procede a disminuír muy lentamente y en forma gradual la dosis de prednisona hasta terminarla y esto se logra en un tiempo de cuatro meses. Se deja al paciente sin ninguna droga por espacio de dos meses.

Se valora su grado de hipersexualidad en este momento, antes del inicio del antiandrógeno y se encuentra: Una acentuada pedofilia masculina que solo es controlada por las condiciones ambientales externas de la pequeña comunidad en que se vive, $y$ una frecuencia promedio entre 20 y 24 eyaculaciones al mes, con intervalos casi siempre mayores de 24 horas; empleándose para ello el onanismo o las relaciones homosexuales.

Se inicia el tratamiento con acetato de ciproterona a la dosis de $100 \mathrm{mgr}$. diarios por vía oral y solo se observa a las tres semanas el primer cambio consistente en demora de la erección pero con una líbido de igual intensidad. A las seis semanas de iniciado el antiandrógeno las eyaculaciones han descendido de una frecuencia de 5 a 6 por semana a 2 ó 3 en los siete días. A las 10 semanas desaparece casi por completo la eyaculación pero persiste la excitación y la erección y las prácticas de masturbación oscilan en una vez por semana con esporádicas eyaculaciones, mínimas en cantidad. Durante cuatro semanas continúan relaciones homosexuales con excitaciones y erecciones débiles que valoradas por el paciente presentan una intensidad del $500 \%$ de lo inicial y su frecuencia no llega a la cuarta parte del comienzo de la droga sin embargo en una ocasión se presentó excitación con erección y eyaculación. A las 20 semanas se aumenta la dosis a 150 miligramos diarios y durante tres semanas sigue un comportamiento semejante; pero a partir de este tiempo nota que se acentúa el retroceso gradual en su líbido y se presentan dos hechos importantes que son la terminación de la pedofilia en forma total y la falta de in- 
terés por el onanismo, con persistencia en forma moderada de la libido y la excitación con mínima erección homosexual.

De las 24 a las 34 semanas, tomando la dosis de 150 miligramos diarios, su líbido y su actividad sexual entran en un reposo total, sin ningún interés sexual, ni erección ni eyaculación, se siente en una gran tranquilidad y paz interior, con una sensación de bienestar que no había experimentado nunca en su vida.

A la semana 35 se baja la dosis a 100 mgr. diarios y a la semana 38 en forma gradual vuelve a despertarse el interés homosexual $y$ aparece de nuevo la erección sin eyaculación; dos semanas más tarde se presenta una relación homosexual con erección y eyaculación; se sube de nuevo la dosis a 150 miligramos diarios y se vuelve al cabo de tres semanas a encontrar el total reposo sexual con las mismas características descritas de tranquilidad y bienestar observadas entre las semanas 24 y 32 en que se ingirió diariamente la misma dosis y casi continúa hasta la semana 60 en que se termina el suministro de la droga por causas ajenas a la voluntad del médico y del paciente.

A las 76 semanas regresa a control e informa que su comportamiento y actividad sexual ha vuelto a su estado inicial y ello lo angustia. Desea de nuevo encontrar esa calma que obtuvo cuando tomaba la dosis de 150 miligramos diarios, aunque anota que la pedofilia no se ha presentado y que puede trabajar con niños y jóvenes sin traumatismo ni dificultad para desarrollar su labor.

\section{COMENTARIOS}

El estudio de este paciente con un seguimiento de siete años y medio con terapia antiandrogénica de un año y dos meses permite hacer una serie de comentarios que se consideran de importancia.

A) En este caso se encontraban aumentada la androsterona, la androstenolona, la dehidroepiandrosterona y la hidroxiandrosterona que son andrógenos de origen suprarrenal que en otros casos estudiados han demostrado un doble efecto, el aumento de la actividad $\mathrm{s}$ sexual y el comportamiento homosexual como ya ha sido publicado (1). En muchos de los pacientes mencionados este cuadro ha cambiado poniendo la suprarrenal en relativo reposo de su esteroidogenesis androgénica, con dosis de prednisona que han oscilado entre los 5 y los 15 miligramos al día. En este caso la terapia con productos de acción semejante a la cortisona no fue eficaz; se piensa como hipótesis de trabajo, en una sensibilidad aumentada de los receptores hipotalámicos androgénicos.

B) De acuerdo con el concepto de $\mathrm{S}$-inbeck y colaboradores (6), la acc.ón del acetato de ciproterona se produce por un antagonismo competitivo con los andrógenos a nivel de los órganos efectores, $\mathrm{y}$ así las células que son sensibles al influjo de la testosterona $c$ a la dihidrotestosterona pierden esta sensibilidad posiblemente por ligar prioritariamente su proteína transportadora al acetado de ciproterona; ello explicaría como en los órganos efectores androgénicos, a pesar de una dosis exógena de testosterona, esta no revela sus características respuestas cuando se ha tomado previamente en dosis suficiente el acetado de ciproterona y como esta sustancia sintetizada por Wiechert (7) es capaz de controlar en forma parcial o total (de acerudo con la dosis empleada) la acción de los endrógenos en los órganos efectores y centros hipotalámicos no importando para ello el que previamente hayan mostrado una hipersensibilidad androgénica, como sería el caso en el paciente presentado.

C) Dado el posible mecanismo de acción del acetato de cíproterona es lógico pensar que en los pacientes con alteraciones acentuadas de la líbido por hipersensibilidad de los centros hipotalámicos, esta terapia será sustitutiva mientras los niveles de la testosterona 
circulante se mantengan y por consiguiente debería tomarse la droga por un largo tiempo; pero en aquellos casos en que las alteraciones de las relaciones de los diferentes esteroides sexuales solo son transitorias como sucede en el acné juvenil, será solo necesaria una terapia con el acetado de ciproterona como antiandrógeno, por un período no muy prolongado.

D) Es bien sabido que la terapia estrogénica no aumenta la líbido y aun más, que una dosis requerida para obtener una anovulación, frecuentemente la disminuye; se ha explicado esto por razones de cambios en la relación normal de estrógenos-andrógenos, y también ha sido suficientemente descrito en la literatura médica que una terapia androgénica a menudo revela un aumento total o relativo de los andrógenos en la mujer produzcan como uno de sus efectos el aumento de la líbido; esto puede ser beneficioso en algunos o en muchos casos, pero en otros ello puede ocasionar serios problemas que desencadenan desde una ligera angustia hasta severas psicopatías, como a menudo se observa en los celibatos inveluntarios de solteras o viudas jóvenes. En estos casos en que por problemas sociales, económicos o religiosos no pueda haber una respuesta positiva a la líbido existente; que está causando por ello trastornos en la esfera psíquica, se considera que el acetato de ciproterona es una terapia eficaz para solucionar estos conflictos del comportamiento sexual humano y ello es también válido para este tipo de problemas en el sexo masculino.

E) En los pacientes con transvertismo, de masculino o femenino (en los casos considerados como una inversión sexoestética) en que una de las primeras etapa del tratamiento es la emasculación que de por sí es irreversible, debe hacerse hoy en día, conociendo la acción del acetato de ciproterona, una terapia con esta droga como etapa previa a la estirpación de las gonadas y si es del caso frenando la esteroideogenesis andrógena con cortisona, para conocer así, tanto el médico como el paciente, la respuesta que se obtendria con el procedimiento quirúrgico y si en verdad es ello lo esperado y anhelado; es decir que, con este procedimiento se pueden lograr en forma transitoria y reversible los efectos que se obtendrían con un procedimiento irreversible.

F) Como comentario final se quiere hacer resaltar que ha sido posible con el acetato de diproterona reproducir lo esperado por los autores Laschet U. y Laschet L. (5) y que se comparte su pensamiento de que esta droga ofrece una ayuda eficaz para regular los casos de hipersexualidad.

\section{RESUMEN}

Se presenta un caso de hipersexualidad masculina; se muestran los hallazgos endocrinológicos y los fracasos con terapias denominadas convencionales. Se describe la evolución obtenida con un tratamiento a base de acetato de ciproterona. Se hacen algunos comentarios.

\section{SUMMARY}

A case of masculine hypersexuality is noticed; the endocrinologic foundings and the failures with therapies know as conventional, are shown. T The evolution resulting from a treatment with aprotherone acetate is described. Some comments are stated.

\section{BIBLIOGRAFIA}

1 CARDONA F. "Fraccionamiento Cromatográfico de los 17 cetoesteroides en le homosexualismo". Primer simposio Esteroides Sexuales de Bogotá. Pág. 28-30. Saladruck. Berlin. 1969. 
2 CARDONA F. "Hallazgos Endocrinológicos y clínicos el Homosexualismo". Rev. Col. Endocrinología. Vol. X, No. 1, diciembre, PPag. 43-49. 1974.

3 DINGENANSE E., y col.: Clinical method for chromatographic colorimetric determination of urinary 17 ketosteroids: Normal adults. J. Clin. Endocr. Mateb. 12:66-85, 1952.

4 DREKTER L.L., y col.: Determination of urinary steroids: preparation of pigmentfree extracts and simplified procedure for estimation of total 17 ketosteroids. J. Clin. Endocrinol. 12: 55-665, 1952.

Este producto ha sido denominado en su etapa experimental "SH8.0714" y en su etapa comercial "ANDROCUR" por la SHERING A.G. Berlin - Bergkamen.
5 LASCHET U., LASCHET L.: Antiandrogen Treatment of Hypersexuality or Abnormal Sexuality in men. Pag. 194-197. Simposio Esteroides sexuales. Bogotá 1968. Ed. Ruiz F., Ramírez J., Willomitzer H. Saladruck. Berlin. 1969.

6 STEINBECK H.: Action of Androgen Antagonists. Pag. 170-193. Simposio Esteroides sexuales. Bogotá. 1968. Ed. Ruiz F., Ramírez J., Willomitzer H. Saladruck. Berlin. 1969.

7 WIESHERT R.: Schering Berlin. 1967. 